\title{
Evaluation of response to conventional chemotherapy and radiotherapy by perfusion computed tomography in non- small cell lung cancer (NSCLC)
}

Carmen Trinidad López ${ }^{1 *}$ D, Javier De La Fuente Aguado², Roque Oca Pernas³ , Carlos Delgado Sánchez-Gracián ${ }^{1}$, Eloisa Santos Armentia ${ }^{1}$, Antonio Vaamonde Liste ${ }^{4}$, Raquel Prada González ${ }^{1}$ and Miguel Souto Bayarri ${ }^{5}$

\begin{abstract}
Background: To evaluate changes in perfusion computed tomography (PCT) parameters induced by treatment with conventional chemotherapy (CCT) alone or with CCT and radiation therapy (RT) in patients with non-small cell lung cancer (NSCLC) and to determine whether these changes correlate with response as defined by the response evaluation criteria in solid tumours version 1.1 (RECIST-1.1).

Methods: Fifty-three patients with a histological diagnosis of NSCLC prospectively underwent PCT of the whole tumour, before/after CCT or before/after CCT and RT. Blood flow (BF), blood volume (BV), permeability (PMB), and mean transit time (MTT) were compared before and after treatment and with the response as defined by RECIST-1. 1. The relationship between changes in the perfusion parameters and in tumour size was also evaluated.

Results: PCT parameters decreased after treatment, significantly for BV $(p=0.002)$ and MTT $(p=0.027)$. The 30 patients with partial response had a significant decrease of $21 \%$ for BV $(p=0.006)$ and $17 \%$ for MTT $(p=0.031)$. A non-significant decrease in all perfusion parameters was found in patients with stable disease $(p>0.137)$. In patients with progressive disease, $\mathrm{MTT}$ decreased by $10 \%(p=0.465)$ and the other parameters did not significantly vary $(p>0.809)$. No significant correlation was found between changes in size and PCT parameters $(p>0.145)$.

Conclusions: Treatment of NSCLC with platinum derivatives, with or without RT, induces changes in PCT parameters. Partial response is associated with a significant decrease in BV and MTT, attributable to the effect of the treatment on tumour vascularisation.
\end{abstract}

Keywords: Carcinoma (non-small cell lung), Lung neoplasms, Perfusion imaging, Response evaluation criteria in solid tumours (RECIST), Tomography (x-ray, computed)

\section{Keypoints}

- Perfusion parameters at computed tomography were compared before and after treatment of non-small cell lung cancer.

- Treatment-induced significant changes in perfusion parameters.

\footnotetext{
* Correspondence: carmen@mianet.eu

'Department of Radiology, POVISA Hospital, 5 Salamanca st, 36208 Vigo,

Pontevedra, Spain

Full list of author information is available at the end of the article
}

- Partial response was associated with a significant decrease in blood volume and mean transit time.

- Changes in perfusion parameters were independent of changes in tumour size.

\section{Background}

Lung cancer is a serious health problem because it represents $13-20 \%$ of cancer diagnoses and is the most common cause of death from malignancy worldwide, with a 5 -year survival rate of only $15-20 \%$ [1].

Until recently, the first line of treatment in advanced lung tumours was platinum derivatives, associated or 
not with radiation therapy (RT) on the tumour [2, 3]. Recently, several genomic mutations have been identified, such as those regarding the endothelial growth factor receptor and anaplastic lymphoma kinase that are associated with response to tyrosine kinase inhibitors and crizotinib, respectively, with a marked improvement in response rates and survival $[4,5]$.

In this setting, the assessment of response to treatment based on tumour size using the response evaluation criteria in solid tumours version 1.1 (RECIST-1.1) guidelines [6] presents some disadvantages, such as disregarding any asymmetric growth of the tumour that does not involve changes in the maximum diameter or the presence of cavitation, necrosis, or haemorrhage that increase the size of the tumour. Therefore, the development of new methods and imaging techniques to assess treatment response using parameters other than size is necessary [7-9].

As happened with targeted therapies, both conventional chemotherapy (CCT) and RT decrease tumour vascularisation [10]. It is known that CCT significantly decreases perfusion parameters in different tumours of the liver, oesophagus, and lungs due to the loss of the angiogenic effect of cytokines after cellular death [11, $12]$; RT causes a reduction in the perfusion values because it acts on the vessels, causing microvascular damage [10]. Therefore, when changes in tumour vascularisation are evaluated, we can detect the effects of the treatment more reliably and earlier, thus avoiding prolonged use and toxicity in patients who do not respond, in addition to decreasing economic costs. Perfusion computed tomography (PCT) is an imaging tool that has attracted increasing attention because the parameters obtained can be an indirect reflection of the vascularisation and vascular physiology of the tumour [8-21], and it can be easily incorporated into routine monitoring protocols for lung cancer [22].

As far as we know, there are only few published studies that evaluate the changes in perfusion parameters before and after treatment in patients with non-small cell lung cancer (NSCLC) [23-27]. Three of these studies include different combinations of antiangiogenic drugs [24-26] and two conventional CCT and/or RT protocols $[23,27]$. The results show that PCT parameters are useful to detect early changes caused by antiangiogenic therapy but not by treatment with platinum derivatives.

The objective of our study was to evaluate the changes in PCT parameters induced by cytotoxic CCT and RT and to determine whether these changes can serve to monitor the response to treatment.

\section{Methods}

The authors had full control of all the data and information presented in this manuscript. Written informed consent was obtained from all the patients involved in the study, and the entire study protocol was approved by the Ethics Committee.

\section{Patients}

Between January 2010 and December 2015, patients with a histological diagnosis of lung cancer were evaluated by a multidisciplinary lung tumour committee and selected by a radiologist with more than 10 years of experience in chest imaging for CTP performance. Figure 1 shows a flow chart of patients included in this study.

A total of 183 patients with a histological diagnosis of NSCLC were prospectively enrolled in our study. All of them underwent $\mathrm{PCT}$ before receiving any treatment (baseline study). A total of 53 patients who received treatment with cytotoxic CCT or concomitant CCT and RT underwent a second PCT study following the treatment (control study).

Inclusion criteria were histological diagnosis of NSCLC; absence of previous oncological treatment for this tumour; maximum tumour diameter larger than $2 \mathrm{~cm}$; and first-line treatment with cytotoxic CCT, with or without associated RT. Exclusion criteria were PCT studies of poor technical quality because of movement artefacts, $\mathrm{x}$-ray beam hardening, noise, or inadequate contrast enhancement; tumours difficult to separate from other lesions such as atelectasis, pneumonitis, or lymphangitis; patients who did not continue follow-up at our hospital; complete response or lesion less than $2 \mathrm{~cm}$ in diameter on the first PCT control after treatment; and presence of respiratory artefacts that could not be corrected by automatic movement correction algorithms.

The baseline study was performed taking into account that iodinated contrast had not been administered in the previous $24 \mathrm{~h}$. The control study was conducted during the same examination as the first standard computed tomography control after treatment with CCT, performing the PCT first and subsequently the study of the entire chest. In patients who received RT, the exam was performed 2 weeks after the end of therapy to avoid inflammatory changes that could influence perfusion parameters.

A total of 53 patients participated in this study, including 42 men (79\%) and 11 women (20\%), with an age of $62.4 \pm 9.9$ years (mean \pm standard deviation; range 35-79 years). The mean dose length product (DLP) for PCT was $463.8 \pm 123.3 \mathrm{mGy} \mathrm{cm}$ (mean \pm standard deviation; range 273-713) and the mean effective radiation dose was $6.40 \pm 1.72 \mathrm{mSv}$ (mean \pm standard deviation; range $3.82-9.82)$.

A total of 16 patients were excluded: 6 patients because of poor technical quality (4 with respiratory artefacts; 2 patients with $x$-ray beam hardening artefacts due 


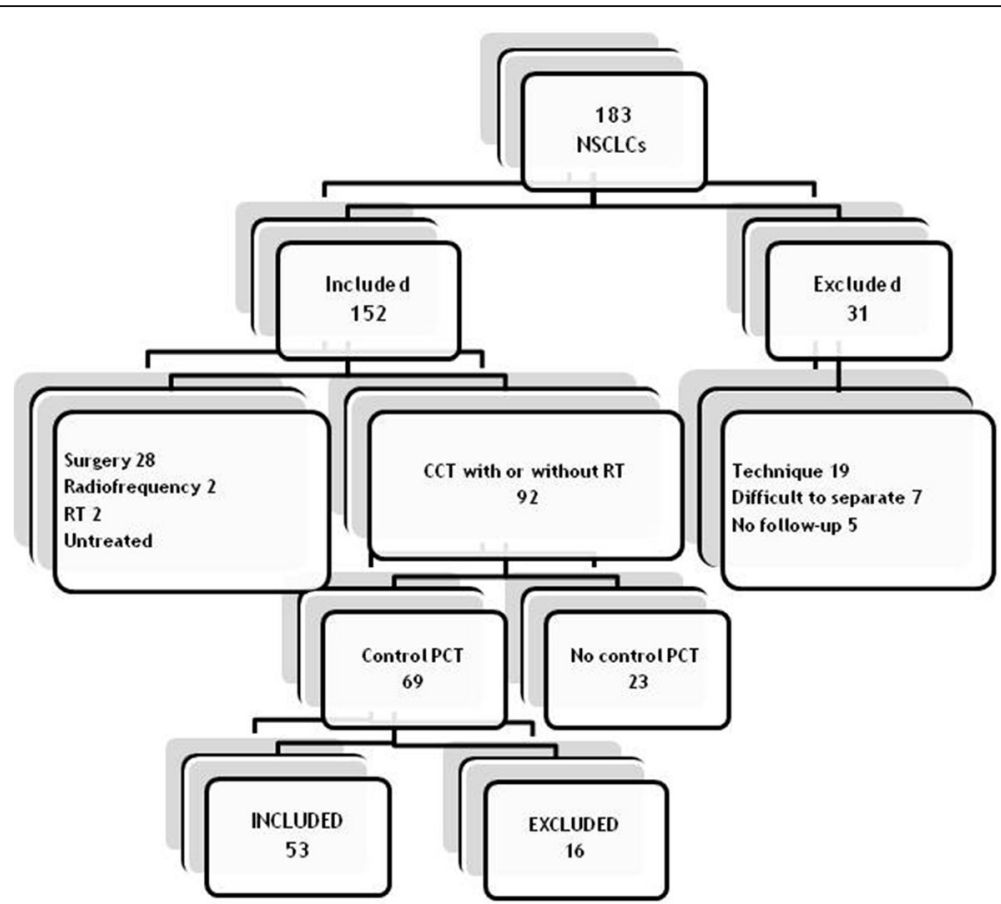

Fig. 1 Study patients flow chart. NSCLC Non-small-celllung cancer, PCT Perfusion computed tomography, CCT Conventional chemotherapy, RT Radiation therapy

to tumour proximity to the superior vena cava and the bone for a tumour located in the pulmonary apex), 7 patients because of the absence of an identifiable tumour or an unmeasurable lesion in the second PCT study, 1 patient due to RT in the previous 2 weeks, and 2 patients in whom the entire tumour volume had not been included in the second study.

The electronic clinical histories were reviewed, and the following data were collected: age, sex, tumour histology, radiological stage at the time of diagnosis according to the TNM classification, type of CCT, association or non-association with concomitant RT, time elapsed in days since the basal PCT study was performed until the first day of CCT, time elapsed in days from the start of the treatment until the second PCT control, and volume and maximum diameter of the tumour in the axial plane before and after treatment.

Two radiologists with 14- and 12-year experience in chest radiology evaluated the first PCT control and classified the patient cases as complete response (CR), partial response $(\mathrm{PR})$, stable disease $(\mathrm{SD})$, or progressive disease (PD) using the RECIST-1.1 criteria [6], applied only to the lesion on which the PCT studies were performed. When there was disagreement, the two readers reached an agreement by consensus.

\section{Patient preparation and PCT technique}

Prior to the exam, the patient was trained to maintain apnoea throughout the study. When the patient was not able to breath-hold, she/he was trained to perform shallow respiration.

A dual-source equipment with 128 rows of detectors (Flash Definition; Siemens, Forchheim, Germany) was used. Once the topogram was performed, a radiologist planned the study field, including the entire lesion along the $z$-axis. When the lesion was not clearly identified in the topogram, an unenhanced scan was performed to locate it.

Fifty $\mathrm{mL}$ of iodinated contrast was injected (Iopromide 300 , Ultravist ${ }^{\circ}$ Bayer Healthcare; Berlin, Germany) at 5 $\mathrm{mL} / \mathrm{s}$, followed by $50 \mathrm{~mL}$ of saline at the same rate.

The PCT acquisition was initiated $2 \mathrm{~s}$ after the injection of the contrast commenced, using the following parameters: $80 \mathrm{kVp}$ and $90 \mathrm{mAs} ; 32 \times 1.2-\mathrm{mm}$ detector configuration; $0.33 \mathrm{~s}$ tube rotation time; 3 or $5 \mathrm{~mm}$ reconstructed image thickness, according to the tumour size; and B20f reconstruction kernel. The total time of the PCT study was always $45 \mathrm{~s}$. The time interval between scans was 1.5 or $1 \mathrm{~s}$, depending on the tumour size along the $z$-axis, which resulted in 30 to 45 scans in each tumour. The total length of the studies along the $z$-axis ranged from 4 to $15 \mathrm{~cm}$.

\section{Post-processing and image analysis}

The data were transferred to a workstation (Multi-Modality Workplace, Siemens, Forchheim, Germany) and processed using the Volume Perfusion Computed Tomography (VPCT) Body program. The PCT studies were 
post-processed and analysed by a senior chest radiologist with 14 years of experience who has received specific training in lung cancer perfusion post-processing, without knowledge of the results of the first standard computed tomography control after treatment with CCT.

First, the automatic motion and noise correction algorithms included in the VPCT Body software were applied. An arterial density-to-time curve was obtained by placing a region of interest in the thoracic aorta, where the unenhanced reference image was selected. The tumour volume was selected via manual segmentation, drawing the contours of the lesion in the axial, coronal, and sagittal planes, using a cut-off threshold of -50-150 $\mathrm{UH}$, which permitted automatically excluding the normal pulmonary parenchyma, non-tumour vascular structures, and calcium, from the segmented volume.

The following perfusion parameters were calculated using a deconvolution model: $\mathrm{BF}$, in $\mathrm{mL} / 100 \mathrm{~mL} / \mathrm{min}$; $\mathrm{BV}$, in $\mathrm{mL} / 100 \mathrm{~mL}$; PMB, in $\mathrm{mL} / 100 \mathrm{~mL} / \mathrm{min}$, and $\mathrm{MTT}$, in seconds. Each parameter was represented as a colour on parametric maps; numerical values were given as mean and standard deviation.

\section{Statistical analysis}

Quantile comparison graphs were used to evaluate if variables followed a normal distribution. All data were normally distributed except BF that was near-normally distributed.

The means of perfusion parameters between responders and no responders as well as between adenocarcinomas and epidermoid carcinomas were compared in baseline study using $t$ test for independent samples. The means and standard deviations of the perfusion parameters were calculated for all of the patients in the baseline study and in the control study and were compared to one another using the $t$ test for paired samples. The perfusion parameters were compared before and after the treatment, at the different response levels according to RECIST-1.1, for all histological subtypes, and in epidermoids and adenocarcinomas separately, using the $t$ test for paired samples. The Pearson correlation coefficient was used to evaluate whether there was a relationship between changes in the perfusion parameters and changes in tumour volume or size.

The programs R ( $\mathrm{R}$ Foundation for Statistical Computing, 2014, V 3.1.0) and SPSS $^{\circ}\left(\mathrm{IBM}^{\circ} \mathrm{SPSS}^{\circ}\right.$ Statistics 20.0.0) were used for the statistical analysis. The level of statistical significance adopted was 0.05 .

\section{Results}

In all cases included, the kinetic model obtained after post-processing and imaging analysis was reliable. $\mathrm{Pa}-$ tient and tumour characteristics are reported in Table 1.
In the first PCT control after the treatment, 30 patients were classified as PR, 20 as $\mathrm{SD}$, and 3 as PD. There were no significant differences between the response groups in terms of age $(p=0.462)$, tumour size $(p=0.559)$, or tumour volume $(p=0.441)$, nor the elapsed time between the baseline perfusion study and the initiation of treatment with chemotherapy $(p=$ 0.803). In the patients with a PR to treatment, the time elapsed between the initiation of CCT treatment and the PCT control study was significantly longer than in the non-responders $(p=0.002)$.

There were no significant differences in perfusion parameters in the baseline study between responders and non-responders: BF 162.00 versus 225.87, $p=0.142$; BV

Table 1 Patients and tumours characteristics $(n=53)$

\begin{tabular}{ll}
\hline Smoking & $48(90.6)$ \\
Family history of lung cancer & $15(28.3)$ \\
Personal history of cancer & $3(5.7)$ \\
Comorbidities & $17(32.1)$ \\
Presentation & $10(18.9)$ \\
Incidental finding & $1(1.9)$ \\
Solitary pulmonary nodule & $50(40.3)$ \\
Cough & $18(34.0)$ \\
Dyspnoea & $13(24.5)$ \\
Pain & $12(22.6)$ \\
Haemoptysis & $27(50.9)$ \\
Constitutional syndrome & \\
Histology & $28(52.8)$ \\
Adenocarcinoma & $16(30.2)$ \\
Epidermoid & $5(9.4)$ \\
Undifferentiated & $3(5.7)$ \\
Large cell & $1(1.9)$ \\
Neuroendocrine &
\end{tabular}

Data are given as absolute frequencies with percentages in parentheses or means \pm standard deviations with ranges in parentheses. CCT Conventional chemotherapy, PCT Perfusion computed tomography, RECIST Response evaluation criteria in solid tumours, $R T$ Radiation therapy 
8.13 versus 9.04, $p=0.42$; PMB 15.48 versus $17.85, p=$ 0.340 ; and TTM 7.05 versus $6.58, p=0.64$. The same was for the response levels according to RECIST-1.1: BF, $p=$ 0.208 ; BV, $p=0.494$; PMB $p=0.587$; and TTM $p=0.727$.

The mean values of all perfusion parameters decreased in the control perfusion study after the treatment, and the difference was significant for BV and MTT (Table 2). The 46 patients treated only with CCT presented a decrease in all perfusion values after treatment, significant for BV $(p=0.030)$ and MTT $(p=0.023)$.

Table 3 shows the changes in the perfusion parameters as a function of the three levels of treatment response according to RECIST-1.1. The patients with PR had a significant decrease of $21 \%$ in BV and of $17 \%$ in MTT. The PMB decreased by $10 \%$ and BF decreased by $5 \%$ (Fig. 2). A non-significant decrease in all perfusion parameters was found in the patients with SD: $15 \%$ for BV, 9\% for MTT, and 17\% for BF; PMB showed practically no variation. The three patients who presented PD had adenocarcinomas. The MTT decreased by $10 \%$, and BV, $\mathrm{BF}$, and PMB did not vary $(p>0.809)$.

Patients who received concomitant treatment with RT presented PR in the first PCT control, and a non-significant decrease was found in all of the perfusion parameters.

No significant correlation was found between the changes in the size and volume of the tumour and the changes in the values of any of the perfusion parameters evaluated in this study $(p \geq 0.220)$.

The most frequent histological types were adenocarcinomas $(n=28)$ and epidermoid carcinomas $(n=16)$. There were no significant differences in perfusion parameters in the baseline study between adenocarcinomas and epidermoid carcinomas: BF 185.73 versus 198.41, $p=0.752$; BV 7.98 versus 7.77, $p=0.848$; $\mathrm{PMB} 17.21$ versus $16.14, p=0.698$; and TTM 6.66 versus $6.05, p=$ 0.560 .

A non-significant decrease in all perfusion parameters in the control study compared to the baseline was observed for the adenocarcinomas (Table 4).

Table 2 Values of perfusion parameters, RECIST-1.1 diameter, and tumour volume in baseline and control perfusion computed tomography $(n=53)$

\begin{tabular}{llll}
\hline & Baseline & Control & $p$ value \\
\hline Blood flow $(\mathrm{mL} / 100 \mathrm{~mL} / \mathrm{min})$ & $189.7 \pm 141.8$ & $170.9 \pm 100.7$ & 0.334 \\
Blood volume $(\mathrm{mL} / 100 \mathrm{~mL})$ & $8.5 \pm 3.9$ & $7.01 \pm 3.4$ & 0.002 \\
Permeability $(\mathrm{mL} / 100 \mathrm{~mL} / \mathrm{min})$ & $16.5 \pm 8.2$ & $15.1 \pm 9.0$ & 0.353 \\
Mean transit time $(\mathrm{s})$ & $6.9 \pm 3.5$ & $5.9 \pm 2.4$ & 0.027 \\
RECIST-1.1 diameter $(\mathrm{mm})$ & $58.2 \pm 23.1$ & $47.0 \pm 21.4$ & $<0.000$ \\
Tumour volume $(\mathrm{cc})$ & $107.1 \pm 138.5$ & $54.6 \pm 83.6$ & $<0.000$ \\
\hline
\end{tabular}

Data are given as means \pm standard deviations with ranges in parentheses. RECIST Response evaluation criteria in solid tumours
The changes in the BV values after the treatment were similar in the patients with PR and SD, showing a decrease by $9 \%$ and $12 \%$, respectively, and were practically unchanged in the patients with PD. The MTT decreased by $4.6 \%$ in the patients with $\mathrm{PR}, 7.5 \%$ in the patients with SD, and $10.4 \%$ in the patients with PD. The PMB decreased by $20 \%$ in the patients with SD, $1.4 \%$ in the patients with PR, and $3 \%$ in the patients with PD.

The epidermoid carcinomas with PR presented a significant decrease by $32 \%$ in BV and by $30 \%$ in MTT. PMB decreased by $30 \%$, approaching the statistical significance $(p=0.062)$. BF presented a non-significant increase of $5 \%$ (Table 5 ).

In the epidermoids with $\mathrm{SD}$, a significant decrease was found in the BF and PMB values, which decreased by $29 \%$ and $59 \%$, respectively, and BV decreased by $19 \%$ $(p=0.269)$. The MTT was the only parameter that increased after treatment, by $48 \%$, although the increase was not significant $(p=0.267)$.

As for the remaining histological subtypes, of the five patients with undifferentiated carcinoma, two presented PR with a decrease in all perfusion parameters after treatment. Of the three patients with SD, one presented a decrease in BF and BV, an unchanged MTT, and an increase in PMB; one presented an increase in BF and PMB and a decrease in BV and MTT; and the third presented an increase in $\mathrm{BF}, \mathrm{BV}$, and $\mathrm{PMB}$ and a decrease in MTT.

Of the three patients with large cell carcinoma, two presented PR with an increase in PMB after treatment and a decrease in BV and MTT, and one presented an increase in BF whereas in another, this parameter decreased. The patient with SD presented an increase in $\mathrm{BF}, \mathrm{BV}$, and PMB and a slight decrease in MTT.

The only patient with a neuroendocrine tumour presented SD with a slight decline in BF, BV, and MTT, and an increase in PMB.

\section{Discussion}

In our study, a decrease was observed in all perfusion parameters after treatment, which, in patients with PR, was significant for BV and MTT in epidermoid tumours, whereas no significant changes were observed in adenocarcinomas. This study suggests a limited capacity of the remaining perfusion parameters for determining the response to treatment. In patients with disease progression following the treatment, the changes were minimal in all NSCLC subtypes.

Because we used the RECIST-1.1 criteria, applying them only to the tumour lesion that was studied with PCT, without taking the remaining targeted lesions into account, it is possible that the changes in perfusion values were related to the change in tumour size. However, we did not detect a relationship between the 
Table 3 Changes in perfusion parameters, RECIST-1.1 diameter, and tumour volume in patients with partial response, stable disease, and progressive disease $(n=53)$

\begin{tabular}{|c|c|c|c|c|c|c|c|c|c|}
\hline & \multicolumn{3}{|c|}{ Partial response $(n=30)$} & \multicolumn{3}{|c|}{ Stable disease $(n=20)$} & \multicolumn{3}{|c|}{ Progressive disease $(n=3)$} \\
\hline & Baseline & Control & $p$ value & Baseline & Control & $p$ value & Baseline & Control & $p$ value \\
\hline Blood flow (mL/100 mL/min) & $162.0 \pm 78.8$ & $154.7 \pm 90.2$ & 0.681 & $234.0 \pm 199.0$ & $195.3 \pm 111.8$ & 0.391 & $171.7 \pm 146.2$ & $170.41 \pm 130.3$ & 0.968 \\
\hline Blood volume (mL/100 mL) & $8.1 \pm 3.1$ & $6.4 \pm 3.0$ & 0.006 & $9.3 \pm 4.9$ & $7.9 \pm 3.7$ & 0.137 & $7.3 \pm 2.8$ & $7.3 \pm 4.8$ & 0.975 \\
\hline Permeability (mL/100 mL/min) & $15.5 \pm 5.7$ & $13.9 \pm 8.3$ & 0.307 & $17.8 \pm 11.3$ & $16.5 \pm 10.2$ & 0.709 & $18.3 \pm 6.5$ & $17.7 \pm 9.6$ & 0.809 \\
\hline Mean transit time (s) & $7.1 \pm 3.1$ & $5.8 \pm 2.4$ & 0.031 & $6.4 \pm 4.1$ & $5.8 \pm 2.3$ & 0.456 & $7.8 \pm 3.5$ & $7.0 \pm 3.6$ & 0.465 \\
\hline RECIST-1.1 diameter (mm) & $59.8 \pm 23.7$ & $41.0 \pm 18.0$ & 0.000 & $55.7 \pm 23.4$ & $53.3 \pm 23.2$ & 0.007 & $58.7 \pm 20.4$ & $65.0 \pm 26.2$ & 0.210 \\
\hline Tumour volume (cc) & $120.2 \pm 154.0$ & $44.6 \pm 59.8$ & 0.000 & $90.4 \pm 122.3$ & $64.3 \pm 108.0$ & 0.006 & $93.3 \pm 73.4$ & $126.7 \pm 97.6$ & 0.145 \\
\hline
\end{tabular}

Significant $p$ values are in bold

RECIST Response evaluation criteria in solid tumours

percent variation of the perfusion parameters and the percent variation of the tumour diameter or volume, which is why these changes are attributable only to the variations in tumour vascularisation induced by treatment. This hypothesis is also supported by the fact that we did not observe changes in perfusion parameters in the baseline study between responders and non-responders [22].

The studies published to date in the literature show highly variable results, as summarised in Table 6. Wang et al. [23] also evaluated changes in the perfusion parameters induced by treatment with RT and CCT with platinum derivatives. However, there are two important differences with respect to our study: The first is that they did not evaluate the entire tumour but rather a size of $20 \mathrm{~mm}$ in the $z$-axis; therefore, in the majority of cases, there was no information about the entire tumour, which is a limitation, particularly when making evaluations before and after the treatment. The second is that they classified the patients into responders and non-responders, considering the tumour mass, which included different target lesions in various organs. Sudarski et al. [27] used the same protocol and did not found significant differences in any of the parameters in NSCLC but in the TTM in SCLC in a series of 100 patients treated with platinum derivatives.

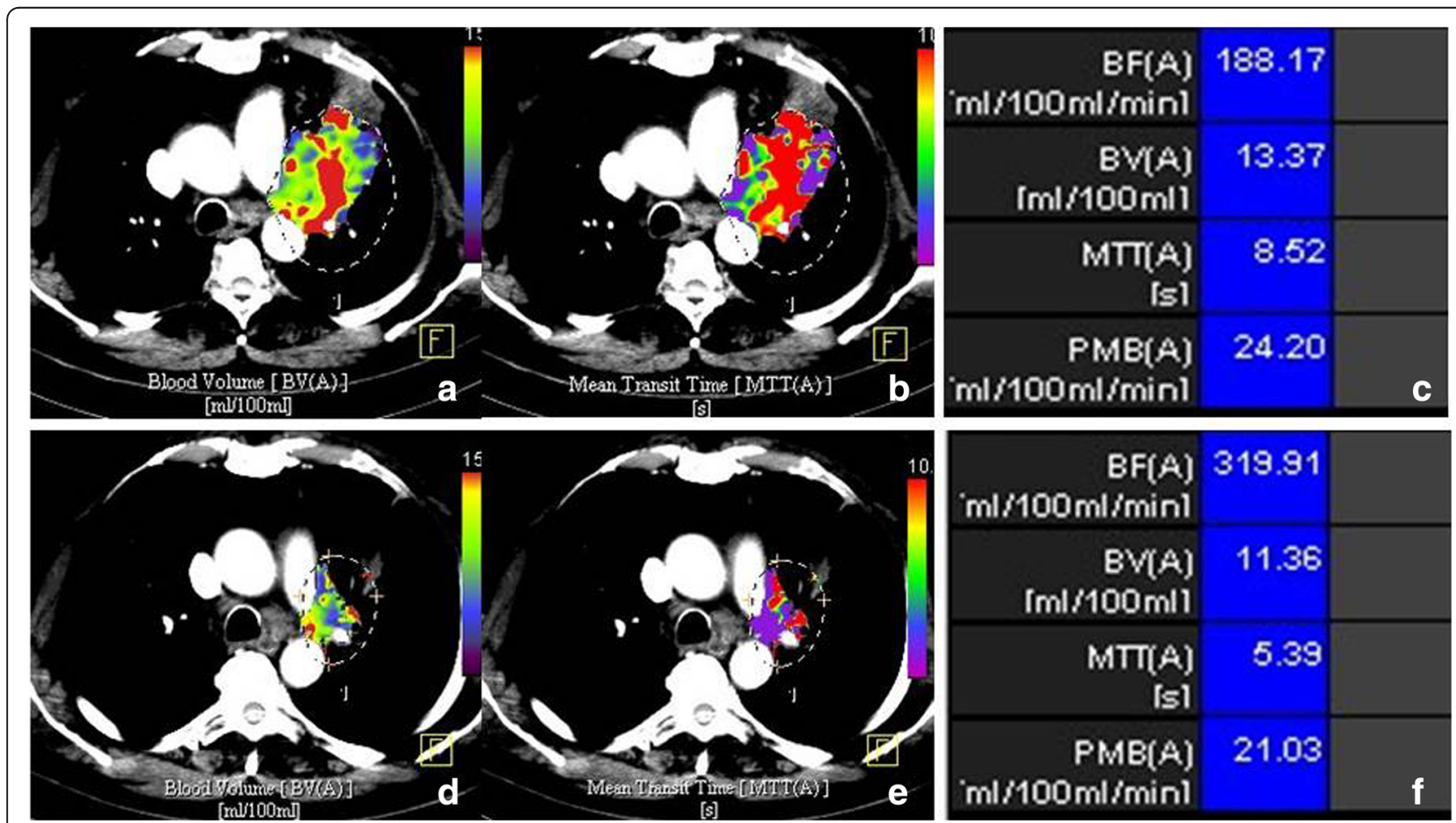

Fig. 2 Perfusion computed tomography of left upper lobe epidermoid carcinoma. Colour coded maps and quantitative values of blood volume and mean transit time before $(\mathbf{a}, \mathbf{b}, \mathbf{c})$ and after $(\mathbf{d}, \mathbf{e}, \mathbf{f})$ platinum derivatives treatment show a decrease in these two perfusion parameters after treatment. BF Blood flow, BV Blood volume, MTT Mean transit time, PMB Permeability 
Table 4 Changes in perfusion parameters, RECIST-1.1 diameter, and tumour volume in adenocarcinomas $(n=28)$

\begin{tabular}{|c|c|c|c|c|c|c|c|c|c|}
\hline & \multicolumn{2}{|c|}{ Partial response $(n=16)$} & \multicolumn{3}{|c|}{ Stable disease $(n=9)$} & \multicolumn{4}{|c|}{ Progressive disease $(n=3)$} \\
\hline & Baseline & Control & $p$ value & Baseline & Control & $p$ value & Baseline & Control & $p$ value \\
\hline Blood flow (mL/100 mL/min) & $157.7 \pm 87.6$ & $139.9 \pm 85.0$ & 0.467 & $240.3 \pm 274.0$ & $192.1 \pm 115.8$ & 0.639 & $171.7 \pm 146.2$ & $170.4 \pm 130.3$ & 0.968 \\
\hline Blood volume (mL/100 mL) & $7.5 \pm 3.3$ & $6.8 \pm 3.0$ & 0.397 & $9.0 \pm 4.9$ & $7.9 \pm 2.8$ & 0.572 & $7.3 \pm 2.8$ & $7.3 \pm 4.8$ & 0.975 \\
\hline Permeability (mL/100 mL/min) & $15.3 \pm 5.6$ & $15.1 \pm 7.7$ & 0.913 & $20.2 \pm 13.9$ & $16.6 \pm 6.1$ & 0.491 & $18.3 \pm 6.5$ & $17.7 \pm 9.6$ & 0.809 \\
\hline Mean transit time $(\mathrm{s})$ & $6.7 \pm 3.25$ & $6.4 \pm 2.3$ & 0.710 & $6.3 \pm 1.6$ & $5.8 \pm 2.0$ & 0.350 & $7.8 \pm 3.5$ & $7.0 \pm 3.6$ & 0.465 \\
\hline RECIST-1.1 diameter (mm) & $58.1 \pm 25.3$ & $41.4 \pm 19.3$ & 0.00 & $54.2 \pm 25.0$ & $51 \pm 24.92$ & 0.041 & $58.7 \pm 20.4$ & $65.0 \pm 26.2$ & 0.210 \\
\hline Tumour volume (cc) & $99.6 \pm 163.9$ & $35.7 \pm 62.9$ & 0.051 & $125.0 \pm 168.4$ & $96.6 \pm 150.0$ & 0.137 & $93.3 \pm 73.4$ & $126.7 \pm 97.6$ & 0.145 \\
\hline
\end{tabular}

Significant $p$ values are in bold

RECIST Response evaluation criteria in solid tumours

Fraioli et al. [24] found a significant decrease in BV and PMB in adenocarcinomas treated with platinum derivatives and bevacizumab. Therefore, their difference in the outcomes could be primarily attributable to the treatment with bevacizumab. PCT better reflects the changes secondary to antiangiogenic therapy, and bevacizumab has been shown to reduce perfusion values in colorectal cancer $[28,29]$. Along the same line, Lind et al. [25] found a significant decline in BF in patients treated with the tyrosine kinase inhibitors sorafenib and erlotinib.

Tacelli et al. [26] concluded that CTP and, in particular, the BV and PMB parameters serve to evaluate the response to treatment with antiangiogenics but not the response to CCT. By contrast, we found a significant decrease in BV in the patients who responded to treatment with platinum derivatives when we analysed all of the histological subtypes and the epidermoid carcinomas, but the decrease in BV was not significant in the adenocarcinomas. In fact, this parameter varied the least at all response levels in this histologic subtype, although there were no differences in baseline perfusion parameters between adenocarcinomas and epidermoid carcinomas. Because the majority of the cases included in the study by Tacelli et al. [26] were adenocarcinomas, the discrepancy in the results may be explained by this difference.

Fraioli et al. [24] and Tacelli et al. [26] found a significant decline in PMB after treatment with antiangiogenics. PMB measures the flow of contrast from the intravascular to the extravascular space and is a reflection of the degree of immaturity of the tumour vessels, which are the main target of antiangiogenic therapy, and therefore, this type of therapy should decrease PMB. The effect of cytotoxic CCT, however, is a decrease in the generation of new vessels; thus, it has a lower impact on PMB and, instead, is expected to decrease parameters such as BF, BV, and MTT.

Fraioli et al. [24] highlighted a discrepancy between the RECIST-1.1 guidelines and the PCT approach, clearly reflected in the behaviour of the perfusion parameters in patients classified with SD. In the 28 adenocarcinomas evaluated in our study, the largest decline in all perfusion parameters occurred in the SD patients, with a significant decline in PMB in the epidermoids. These findings support the discrepancy between PCT and the RECIST-1.1 criteria. Even in the initial stages after treatment, PCT may reflect subtle changes in the biological activity of the tumour that are not translated into macroscopic changes in the tumour size; therefore,

Table 5 Changes in perfusion parameters, RECIST-1.1 diameter, and tumour volume in epidermoid carcinomas $(n=16)$

\begin{tabular}{|c|c|c|c|c|c|c|}
\hline & \multicolumn{3}{|c|}{ Partial response $(n=10)$} & \multicolumn{3}{|c|}{ Stable disease $(n=6)$} \\
\hline & Baseline & Control & $p$ value & Baseline & Control & $p$ value \\
\hline $\begin{array}{l}\text { Blood flow } \\
\text { (mL/100 mL/min) }\end{array}$ & $176.1 \pm 76.1$ & $186.5 \pm 106.4$ & 0.775 & $234.9 \pm 97.5$ & $165.8 \pm 108.7$ & 0.010 \\
\hline Blood volume (mL/100 mL) & $8.6 \pm 3.3$ & $5.9 \pm 2.6$ & 0.007 & $6.4 \pm 2.8$ & $5.2 \pm 3.4$ & 0.269 \\
\hline Permeability (mL/100 mL/min) & $15.9 \pm 6.9$ & $11.1 \pm 7.0$ & 0.062 & $16.5 \pm 11.5$ & $6.7 \pm 4.1$ & 0.036 \\
\hline Mean transit time $(\mathrm{s})$ & $7.5 \pm 3.6$ & $5.3 \pm 2.7$ & 0.018 & $3.6 \pm 1.7$ & $5.3 \pm 2.9$ & 0.267 \\
\hline RECIST-1.1 diameter (mm) & $54.5 \pm 21.4$ & $35.7 \pm 15.7$ & 0.002 & $62.5 \pm 24.3$ & $59.8 \pm 23.9$ & 0.082 \\
\hline Tumour volume (cc) & $142.5 \pm 171.8$ & $56.1 \pm 62.6$ & 0.038 & $57.0 \pm 4.6$ & $22.5 \pm 18.9$ & 0.021 \\
\hline
\end{tabular}

RECIST Response evaluation criteria in solid tumours

Significant $p$ values are in bold 
Table 6 Comparison between the published studies and the current study

\begin{tabular}{|c|c|c|c|c|c|c|c|c|c|c|}
\hline $\begin{array}{l}\text { First author } \\
\text { [reference] }\end{array}$ & $\begin{array}{l}\text { Number of } \\
\text { patients }\end{array}$ & Histology & CCT & RT & $\begin{array}{l}\text { Targeted } \\
\text { therapy }\end{array}$ & $\begin{array}{l}\text { RECIST } \\
\text { assessment }\end{array}$ & $\begin{array}{l}\text { Blood } \\
\text { flow }\end{array}$ & $\begin{array}{l}\text { Blood } \\
\text { volume }\end{array}$ & $\begin{array}{l}\text { Mean transit } \\
\text { time }\end{array}$ & Permeability \\
\hline Wang et al [23] & 35 & $\begin{array}{l}\text { Epidermoid } \\
\text { Adenocarcinoma }\end{array}$ & No & Yes & No & Response & $\downarrow$ & $\downarrow$ & $\uparrow$ & $\begin{array}{l}\downarrow \\
p>0.05\end{array}$ \\
\hline Wang et al [23] & 12 & $\begin{array}{l}\text { Epidermoid } \\
\text { Adenocarcinoma }\end{array}$ & Yes & No & No & $\begin{array}{l}\text { Response } \\
\text { Progression }\end{array}$ & $\uparrow$ & $\uparrow$ & $\uparrow$ & $\uparrow$ \\
\hline Fraioli et al [24] & 45 & Adenocarcinoma & Yes & & Bevacizumab & Response & & $\downarrow$ & & $\downarrow$ \\
\hline Lind et al [25] & 23 & $\begin{array}{l}\text { Adenocarcinoma } \\
\text { Epidermoid } \\
\text { Large cell }\end{array}$ & & & $\begin{array}{l}\text { Sorafenib } \\
\text { Erlotinib }\end{array}$ & Response & $\downarrow$ & & & \\
\hline Tacelli et al [26] & 17 & Adenocarcinoma & Yes & & Bevacizumab & Response & & $\downarrow$ & $\downarrow$ & \\
\hline Tacelli et al [26] & 23 & $\begin{array}{l}\text { Adenocarcinoma } \\
\text { Epidermoid } \\
\text { Large cell }\end{array}$ & Yes & No & No & Response & & $\leftrightarrow$ & $\leftrightarrow$ & \\
\hline Sudarski et al [27] & 100 & $\begin{array}{l}\text { Adenocarcinoma } \\
\text { Epidermoid }\end{array}$ & Yes & No & No & $\begin{array}{l}\text { Response } \\
\text { Progression Stable }\end{array}$ & $\leftrightarrow$ & $\leftrightarrow$ & $\leftrightarrow$ & $\leftrightarrow$ \\
\hline Sudarski et al [27] & 100 & Small cell & Yes & No & No & $\begin{array}{l}\text { Response } \\
\text { Progression Stable }\end{array}$ & $\leftrightarrow$ & $\leftrightarrow$ & $\downarrow$ & $\leftrightarrow$ \\
\hline $\begin{array}{l}\text { Trinidad et al } \\
\text { [current study] }\end{array}$ & 7 & $\begin{array}{l}\text { Adenocarcinoma } \\
\text { Epidermoid Large cell }\end{array}$ & Yes & Yes & No & Response & $\begin{array}{l}\downarrow \\
p>0.05\end{array}$ & $\begin{array}{l}\downarrow \\
p>0.05\end{array}$ & $\begin{array}{l}\downarrow \\
p>0.05\end{array}$ & $\begin{array}{l}\downarrow \\
p>0.05\end{array}$ \\
\hline $\begin{array}{l}\text { Trinidad et al } \\
\text { [current study] }\end{array}$ & 46 & $\begin{array}{l}\text { Adenocarcinoma } \\
\text { Epidermoid Large cell }\end{array}$ & Yes & No & No & Response & & $\downarrow$ & $\downarrow$ & \\
\hline
\end{tabular}

Changes by treatment in perfusion parameters in lung cancer reported by different published studies: $\uparrow=$ significant increase; $\downarrow=$ significant decrease; $\uparrow=$ in some patients, the parameter increases and in other patients, it decreases; $\leftrightarrow=$ there were no changes; $p>0.05$ nonsignificant changes

RECIST Response evaluation criteria in solid tumours

it may be more useful than the conventional size evaluated with RECIST-1.1.

There are studies of different lung cancer tumours that evaluated the changes in perfusion parameters following treatment with CCT and RT, with widely varying results [29-31]. These results suggest that PCT in lung cancer serves to monitor the response to treatment with targeted therapies, but its usefulness in treatment with cytotoxic RT and CCT is unclear. However, in our study the perfusion parameters, particularly BV and MTT, changed after these treatments and these changes appeared to be attributable solely to the treatment.

Taking into account that the majority of patients with advanced lung cancer receive platinum derivatives and $\mathrm{RT}$ as a first-line treatment protocol and the limitations of the response criteria based solely on tumour size (as are those of RECIST-1.1), PCT may have a role in monitoring treatment response. Despite the observed decrease in BV and MTT in patients with PR, larger studies are necessary to determine the usefulness of these parameters in predicting the degree of responsiveness and incorporating it into monitoring protocols, specifying what histologic subtype would most benefit from the quantitative analysis of the perfusion and defining the time intervals for control studies.

Our study has some limitations. The time elapsed between the baseline perfusion study and the initiation of treatment with CCT was variable. In patients with PR, the time elapsed between the initiation of treatment with $\mathrm{CCT}$ and the control perfusion study was significantly longer than in the non-responders. Although this finding could have affected the results, we believe that it was minimal because PCT was performed on the same day as the first standard computed tomography control and, therefore, the level of response reported in clinical practice did not vary. The same acquisition parameters and contrast dose were used, without taking the body mass index or tumour size into account, to meet the requirements of the kinetic model for calculating the perfusion values. Finally, we opted to apply the RECIST-1.1 criteria only to the tumour on which the perfusion study was performed because the perfusion values of the primary lesion cannot be extrapolated to the remaining lesions, and metastatic lesions may respond to the treatment differently than the primary tumour. Additionally, in the tumours treated with RT, this therapy only acts on the targeted lesion. 
In conclusion, treatment with platinum derivatives, with or without RT, induces changes in PCT parameters. Except in the adenocarcinomas, PR is associated with a significant decrease in BV and MTT, attributable to the effect of the treatment on tumour vascularisation. Patients with no significant changes were obtained in any of the parameters.

\section{Abbreviations \\ BF: Blood flow; BV: Blood volume; CCT: Conventional chemotherapy; CR: Complete response; MTT: Mean transit time; NSCLC: Non-small cell lung cancer; PCT: Perfusion computed tomography; PD: Progressive disease; PMB: Permeability; PR: Partial response; RECIST: Response evaluation criteria in solid tumours; RT: Radiation therapy; SD: Stable disease; VPCT: Volume perfusion computed tomography}

\section{Funding}

There is no source of funding for this work.

\section{Availability of data and materials}

The datasets used and/or analysed during the current study are available from the corresponding author on reasonable request.

\section{Authors' contributions}

Responsible for the integrity of the study: CTL. Study design: CTL. Data collection: CTL, CDS. Analysis and interpretation of data: CTL, AVL, ROP, JDA Statistical treatment: CTL, AVL. Bibliographic search: CTL. Work writing: CTL, ROP, JDA. Critical review of the manuscript with intellectually relevant contributions: CTL, ROP, JDA, CDS, ESA, RPG, MSB. Approval of the final version: $C T L, A V L, J D A, R O P, C D S, E S A, R P G$

\section{Ethics approval and consent to participate}

Approved by the autonomic Committee of Research Ethics of Galicia (Spain). Registration Code: 2016/004.

\section{Consent for publication}

Not applicable

\section{Competing interests}

The authors declare that they have no competing interests.

\section{Publisher's Note}

Springer Nature remains neutral with regard to jurisdictional claims in published maps and institutional affiliations.

\section{Author details \\ 'Department of Radiology, POVISA Hospital, 5 Salamanca st, 36208 Vigo, Pontevedra, Spain. ${ }^{2}$ Department of Internal Medicine, POVISA Hospital, Vigo, Spain. ${ }^{3}$ Department of Radiology, Osatek, Urduliz Hospital, Vizcaya, Spain. ${ }^{4}$ Department of Statistics and Operational Research, Faculty of Economic and Business Sciences, Vigo University Spain, Vigo, Spain. ${ }^{5}$ Department of Radiology, Complexo Hospitalario Universitario de Santiago de Compostela, Santiago de Compostela, Spain.}

Received: 30 January 2019 Accepted: 2 May 2019

Published online: 13 June 2019

\section{References}

1. Key statistics for lung cancer. American Cancer Society web site. Available via https://www.cancer.org/cancer/non-small-cell-lung-cancer/about/keystatistics.html. Accessed Feb 2017

2. National Comprehensive Cancer Network Clinical Practice Guidelines in Oncology, Non-Small Cell Lung Cancer, version 1. 2015. Available via https://www.nccn.org/professionals/physician_gls/\#site. Accessed Feb 2019

3. Breathnach OS, Freidlin B, Conley B et al (2001) Twenty-two years of phase III trials for patients with advanced non-small-cell lung cancer: sobering results. J Clin Oncol 19:1734-1742. https://doi.org/10.1200/JCO. 2001.19.6.1734
4. Choi CM, Kim MY, Lee JC, Kim HJ (2014) Advanced lung adenocarcinoma epidermal growth factor receptor : CT findings after tyrosine kinase inhibitor therapy. Radiology 270:574-582. https://doi.org/10.1148/radiol.13121824

5. Ebos JM, Kerbel RS (2011) Antiangiogenic therapy: impact on invasion, disease progression, and metástasis. Nat Rev Clin Oncol 8:210-221. https:// doi.org/10.1038/nrclinonc.2011.21

6. Eisenhauer EA, Therasse P, Bogaerts J et al (2009) New response evaluation criteria in solid tumours: revised RECIST guideline (version1.1). Eur J Cancer 45:228-247. https://doi.org/10.1016/j.ejca.2008.10.026

7. Nishino M, Hatabu H, Johnson BE, Mc Loud TC (2014) State of the art: response assessment in lung cancer in the era of genomic medicine. Radiology 271:6-27. https://doi.org/10.1148/radiol.14122524

8. Garcia Figueiras R, Padhani AR, Beer AJ et al (2015) Imaging of tumor angiogenesis for radiologists (part 1: biological and technical basis). Curr Probl Diagn Radiol 44:425-436. https://doi.org/10.1067/j.cpradiol.2015.02.010

9. Nishino M, Jagannathan JP, Krajewski KM et al (2012) Personalized tumor response assessment in the era of molecular medicine: cancer-specific and therapy-specific response criteria to complement pitfalls of RECIST. AJR Am J Roentgenol 198:737-745. https://doi.org/10.2214/AJR.11.7483

10. Baeten $\mathrm{Cl}$, Castermans K, Lammering $\mathrm{G}$ et al (2006) Effects of radiotherapy and chemotherapy on angiogenesis and leukocyte infiltration in rectal cancer. Int J Radiat Oncol Biol Phys 66:1219-1227. https://doi.org/10.1016/j. ijrobp.2006.07.1362

11. Zhang Q, Yuan ZG, Wang DQ, Yan ZH, Tang J, Liu ZQ (2010) Perfusion CT findings in liver of patients with tumor during chemotherapy. World J Gastroenterol 16:3202-3205. https://doi.org/10.3748/wjg.v16.i25.3202

12. Kiessling F, Boese J, Corvinus C et al (2004) Perfusion CT in patients with advanced bronchial carcinomas: a novel chance for characterization and treatment monitoring? Eur Radiol 14:1226-1233. https://doi.org/10.1007/ s00330-004-2288-2

13. lagaru A, Gambhir S (2013) Imaging tumor angiogenesis: the road to clinical utility. AJR Am J Roentgenol 201:W183-W191. https://doi.org/10.2214/AJR. 12.8568

14. García-Fiqueiras R, Goh VJ, Padhani AR et al (2013) CT perfusion in oncologic imaging: a useful tool? AJR Am J Roentgenol 200:8-19. https:// doi.org/10.2214/AJR.11.8476

15. Miles KA, Lee TY, Goh V et al (2012) Current status and guidelines for the assessment of tumour vascular support with dynamic contrast-enhanced computed tomography. Eur Radiol 22:1430-1441. https://doi.org/10.1007/ s00330-012-2379-4

16. Henzler T, Goldstraw P, Wenz F et al (2015) Perspectives of novel imaging techniques for staging, therapy response assessment, and monitoring of surveillance in lung cancer. J Thorac Oncol 10:237-249. https://doi.org/10. 1097/JTO.0000000000000412

17. Kim H, Park CM, Goo JM, Wildberger JE, Kauczor HU (2015) Quantitative computed tomography imaging biomarkers in the diagnosis and management of lung cancer. Invest Radiol 50:571-583. https://doi.org/10. 1097/RLI.0000000000000152

18. Li Y, Yang ZG, Chen TW, Sun JY, Lu YR (2008) Peripheral lung carcinoma: correlation of angiogenesis and first-pass perfusion parameters of 64 detector row CT. Lung Cancer 61:44-53. https://doi.org/10.1016/j.lungcan. 2007.10.021

19. Tacelli N, Remy-Jardin M, Copin MC et al (2010) Assessment of non-small cell lung cancer perfusion: pathologic-CT correlation in 15 patients. Radiology 257:863-871. https://doi.org/10.1148/radiol.10100181

20. Spira D, Neumeister H, Spira SM et al (2013) Assessment of tumor vascularity in lung cancer using volume perfusion CT (VPCT) with histopathologic comparison: a further step toward an individualized tumor characterization. J Comput Assist Tomogr 37:15-21. https://doi.org/10.1097/ RCT.0b013e318277c84f

21. Kambadakone AR, Sahani DV (2009) Body perfusion CT: technique, clinical applications, and advances. Radiol Clin North Am 47:161-178. https://doi. org/10.1016/j.rcl.2008.11.003

22. Trinidad López C, Souto Bayarri M, Oca Pernas R et al (2018) Characteristics of computed tomography perfusion parameters in non-small-cell-lungcancer and its relationship to histology, size, stage an treatment response. Clin Imaging 50:5-12. https://doi.org/10.1016/j.clinimag.2017.12.001

23. Wang J, Wu N, Cham M, Song Y (2009) Tumor response in patients with advanced non-small cell lung cancer: perfusion CT evaluation of chemotherapy and radiation therapy. AJR Am J Roentgenol 193:1090-1096. https://doi.org/10.2214/AJR.08.1367 
24. Fraioli F, Anzidei M, Zaccagna $F$ et al (2011) Whole-tumor perfusion CT in patients with advanced lung adenocarcinoma treated with conventional and antiangiogenetic chemotherapy: initial experience. Radiology 259:574-582. https://doi.org/10.1148/radiol.11100600

25. Lind JS, Meijerink MR, Dingemans AM et al (2010) Dynamic contrast enhanced $C T$ in patients treated with sorafenib and erlotinib for non-small cell lung cancer: a new method of monitoring treatment? Eur Radiol 20:2890-2898. https://doi.org/10.1007/s00330-010-1869-5

26. Tacelli N, Santangelo T, Scherpereel A et al (2013) Perfusion CT allows prediction of therapy response in non-small cell lung cancer treated with conventional and anti-angiogenic chemotherapy. Eur Radiol 23:2127-2136. https://doi.org/10.1007/s00330-013-2821-2

27. Sudarski S, Shi J, Schmid-Bindert G et al (2015) Dynamic volume perfusion CT parameters versus RECIST for the prediction of outcome in lung cancer patients treated with conventional chemotherapy. J Thorac Oncol 10:164-171. https://doi.org/10.1097/JT0.0000000000000376

28. Koukourakis Ml, Mavanis I, Kouklakis G et al (2007) Early antivascular effects of bevacizumab anti-VEGF monoclonal antibody on colorectal carcinomas assessed with functional CT imaging. Am J Clin Oncol 30:315-318. https:// doi.org/10.1097/01.coc.0000258119.90805.ca

29. Bellomi M, Petralia G, Sonzogni A, Zampino MG, Rocca A (2007) CT perfusion for the monitoring of neoadjuvant chemotherapy and radiation therapy in rectal carcinoma: initial experience. Radiology 244:486-493. https://doi.org/10.1148/radiol.2442061189

30. Sahani DV, Kalva SP, Hamberg LM et al (2005) Assessing tumor perfusion and treatment response in rectal cancer with multisection $\mathrm{CT}$ : initial observations. Radiology 234:785-792. https://doi.org/10.1148/radiol. 2343040286

31. Park MS, Klotz E, Kim MJ et al (2009) Perfusion CT: noninvasive surrogate marker for stratifi cation of pancreatic cancer response to concurrent chemo and radiation therapy. Radiology 250:110-117. https://doi.org/10 1148/radiol.2493080226

\section{Submit your manuscript to a SpringerOpen ${ }^{\circ}$ journal and benefit from:}

- Convenient online submission

- Rigorous peer review

- Open access: articles freely available online

- High visibility within the field

- Retaining the copyright to your article

Submit your next manuscript at $\boldsymbol{\nabla}$ springeropen.com 